\title{
0.5-Gb/s OFDM-Based Laser Data and Power Transfer Using a GaAs Photovoltaic Cell
}

\author{
John Fakidis, Member, IEEE, Stefan Videv, Henning Helmers, and Harald Haas, Fellow, IEEE
}

\begin{abstract}
In this letter, we demonstrate for the first time the additional capability of high-speed data communication for single-junction photovoltaic (PV) cells. A record 3-dB bandwidth of $24.5 \mathrm{MHz}$ is reported for a gallium arsenide (GaAs) PV cell. The PV cell is shown to achieve a power efficiency of at least $42 \%$ when irradiance of $0.46 \mathrm{~W} / \mathrm{cm}^{2}$ is received from $847-\mathrm{nm}$ verticalcavity surface-emitted laser. Optimized bit-and-power-loaded optical orthogonal frequency-division multiplexing (OFDM) is applied to use the communication bandwidth most efficiently. With this, a data rate of $0.5 \mathrm{~Gb} / \mathrm{s}$ is achieved for a 2-m OFDMbased laser link. To the best of our knowledge, the reported data rates achieved with a GaAs PV cell as the detector are the highest for simultaneous optical wireless information and power transfer.
\end{abstract}

Index Terms-Energy harvesting, gallium arsenide, OFDM, photovoltaic cells, vertical cavity surface emitting lasers

\section{INTRODUCTION}

$\mathbf{T}$ HE concept of simultaneous wireless information and power transfer (SWIPT) has been widely acknowledged as one of the main enablers of the 'Internet-of-Things' era [1]. While the vast majority of research has focused on the use of radio-frequency (RF) signals [2], only a few studies consider the optical region of the electromagnetic spectrum for data transfer and energy harvesting (EH) [3]-[8]. Sun provides irradiance levels on the Earth's surface ranging from tens to more than a hundred of $\mathrm{mW} / \mathrm{cm}^{2}$ [9]; these irradiance values are two orders of magnitude higher than those of ambient RF radiation [10]. Therefore, there is a unique potential to exploit the already existing infrastructure of photovoltaic (PV) cells for (a) $\mathrm{EH}$ from sunlight and (b) $\mathrm{EH}$ with simultaneous data communication from laser or LED-based radiation.

A typical example of a PV receiver designed for SWIPT consists of two separate branches for power splitting [6]: a large inductor acting as an ac choke and a resistor are used to pass the dc component; and a large capacitor acting as a dc open and a resistor are used to transfer the ac part of the signal. Since sunlight is considered to be constant over the typical duration of a data symbol, it contributes only to the dc power generation without affecting the ac communication signal. It is shown in [11] that sunlight has an insignificant effect on the information signal modulated using optical orthogonal frequency-division multiplexing (OFDM).

The work of $\mathrm{H}$. Haas was supported by the Engineering and Physical Sciences Research Council under the Established Career Fellowship Grant $\mathrm{EP} / \mathrm{K} 008757 / 1$.

J. Fakidis, S. Videv and H. Haas are with the School of Engineering, Institute for Digital Communications, Li-Fi Research and Development Center, University of Edinburgh, Edinburgh EH9 3JL, U.K. (e-mail: j.fakidis@ed.ac.uk; s.videv@ed.ac.uk; h.haas@ed.ac.uk).

H. Helmers is with the Fraunhofer Institute for Solar Energy Systems (ISE), 79110 Freiburg, Germany (e-mail: henning.helmers@ise.fraunhofer.de).
The application of SWIPT in the optical spectrum was only recently explored independently in [3] and [4] using a white LED and silicon ( $\mathrm{Si}$ ) solar cells. The $3-\mathrm{dB}$ bandwidth of $10 \mathrm{kHz}$ and the data rate of $3 \mathrm{~kb} / \mathrm{s}$ achieved in [3] are considered to be insufficient for high-speed data communication. However, received data rates of $7 \mathrm{Mb} / \mathrm{s}$ and $2.1 \mathrm{~mW}$ of harvested power from a single multi-crystalline Si solar panel are reported in [4] for the first time. Following on from these initial demonstrations, a high-speed optical wireless (OW) link was created in [6] using a white LED and the same solar panel with that used in [4]. While the $3-\mathrm{dB}$ bandwidth of the PV panel is shown to be up to $100 \mathrm{kHz}$, the use of optical OFDM results in a boosted data rate of $12 \mathrm{Mb} / \mathrm{s}$ [6]. The use of organic solar cells for high-speed data reception is shown for the first time in [5] with a reported 3-dB bandwidth of 1.3 MHz. Again, the application of dc optical OFDM gives a link data rate of $34.2 \mathrm{Mb} / \mathrm{s}$ with a respective bit error rate (BER) of $4.1 \times 10^{-4}$ [5]. Laser power converters (LPCs) are a particular type of single-junction PV cells that are able to achieve maximum values of power efficiency under the conditions of monochromatic illumination [12]. A record power efficiency of $58.2 \%$ has been reported by researchers in the Fraunhofer ISE using a gallium arsenide (GaAs) LPC under $809-\mathrm{nm}$ laser of $110-\mathrm{W} / \mathrm{cm}^{2}$ irradiance [12].

The application of a reverse bias to a solar panel is shown to increase its $3-\mathrm{dB}$ bandwidth from $90 \mathrm{kHz}$ to $120 \mathrm{kHz}$ in [13]. This is attributed to the reduced drift time of the photocarriers inside the depletion region of the PV cells. At the same time, the width of the depletion region increases resulting in a higher responsivity of the PV panel [13]. However, the PV efficiency is expected to be reduced because of the additional electrical power consumption of the reverse bias.

In this letter, the $\mathrm{EH}$ and communication capabilities of a circular GaAs PV cell with a 1-mm diameter are investigated. Since the optimal region of operation wavelengths for GaAs LPCs is between $800 \mathrm{~nm}$ and $850 \mathrm{~nm}$ [12], a high-speed 847$\mathrm{nm}$ vertical-cavity surface-emitting laser (VCSEL) is used at the transmitter. The receiver comprises the PV cell and a variable resistor. Our objective is to determine the optimum resistor values for $\mathrm{EH}$ and data communication independently. Future work will consider the development of an optimal PV receiver for SWIPT. Each of the two branches will include the resistor that maximizes the harvested power and 3-dB bandwidth of the cell, respectively. The frequency response of the PV cell is characterized for various resistance values; thus, a record $3-\mathrm{dB}$ bandwidth of $24.5 \mathrm{MHz}$ is reported. The harvested electrical power of the PV receiver is also determined as a function of the load resistance; a high conversion efficiency of at least $42 \%$ is achieved for the LPC under $0.46-\mathrm{W} / \mathrm{cm}^{2}$ 
irradiance of 847-nm laser. Optical OFDM with bit and power loading is applied for the optimal use of the non-flat frequency response of the communication channel; thus, a record data rate of $0.5 \mathrm{~Gb} / \mathrm{s}$ is reported for a $1-\mathrm{mm}$ and $2-\mathrm{m}$ OW link.

The rest of the letter is organized as follows: the frequency response and harvested power of the LPC are given in Sec. II. In Sec. III, the data communication performance is given. Finally, concluding remarks are provided in Sec. IV.

\section{FREQUENCY RESPONSE AND HARVESTED POWER}

In this section, a physical model of the PV cell for $\mathrm{EH}$ and the experimental setup for information and power transfer are given. In order to validate the experimental data of the power efficiency of the PV cell, an optimization problem is solved to determine five unknown parameters of the analytical model. The estimated parameters are used for curve fitting of the measured data.

\section{A. Physical model of the PV-cell-based receiver for $E H$}

The traditional single-diode physical equivalent dc circuit of the PV cell is considered [6], [9]. This model contains five unknown parameters, i.e. $I_{0}, R_{\mathrm{s}}, n, R_{\mathrm{sh}}$, and $I_{\mathrm{ph}}$. Quantities $I_{0}$ and $I_{\mathrm{ph}}$ denote the dark saturation current of the diode and the photo-current of the PV cell, respectively; $R_{\mathrm{s}}$ and $R_{\text {sh }}$ represent the series and shunt resistance, respectively; and $n$ is the ideality factor of the diode. The load resistor $R_{1}$ is varied to measure the modified load voltage $V_{1}$ and current $I_{1}$. Therefore, the harvested electrical power is calculated by using $P_{\mathrm{Rx}, \text { elec }}=V_{1} I_{\mathrm{l}}$. The unknown parameters of the model are estimated using an exhaustive search algorithm with discrete search space. Hence, the optimization problem that is solved is expressed by [6]:

$$
\begin{aligned}
& \min _{I_{0}, R_{\mathrm{s}}, n, R_{\mathrm{sh}}, I_{\mathrm{ph}}} \sum_{i=1}^{N_{\mathrm{m}}} \mid I_{0}\left\{\exp \left\{\frac{q\left[V_{1}(i)+R_{\mathrm{S}} I_{1}(i)\right]}{n k_{\mathrm{B}} T}\right\}-1\right\} \\
& +\frac{V_{\mathrm{l}}(i)+R_{\mathrm{s}} I_{1}(i)}{R_{\mathrm{sh}}}-I_{\mathrm{ph}}+\left.I_{\mathrm{l}}(i)\right|^{2} \\
& \text { subject to : } \quad I_{0, \min } \leq I_{0} \leq I_{0, \max } \\
& R_{\mathrm{s}, \text { min }} \leq R_{\mathrm{s}} \leq R_{\mathrm{s}, \text { max }} \\
& n_{\text {min }} \leq n \leq n_{\text {max }} \\
& R_{\text {sh,min }} \leq R_{\text {sh }} \leq R_{\text {sh,max }} \\
& I_{\mathrm{ph}, \text { min }} \leq I_{\mathrm{ph}} \leq I_{\mathrm{ph}, \text { max }},
\end{aligned}
$$

where $N_{\mathrm{m}}$ is the number of the measured data; $q=1.6 \times$ $10^{-19} \mathrm{C}$ and $k_{\mathrm{B}}=1.38 \times 10^{-23} \mathrm{~J} / \mathrm{K}$ denote the electron's charge and Boltzmann constant, respectively; and $T=296 \mathrm{~K}$ is the measured temperature of the PV cell - this is very close to the temperature of $25^{\circ} \mathrm{C}$ used at standard test conditions. The current $I_{\mathrm{d}}$ of the junction diode is given as a function of $I_{0}$ and $n$ by the first term of the sum in (1). Also, the subscripts min and max refer to the minimum and maximum values of the parameters given as input to the algorithm, respectively.

\section{B. Experimental setup}

The frequency response of the PV cell is determined using the experimental setup shown in Fig. 1. The harvested power of the LPC is measured using the three first blocks of Fig. 1.

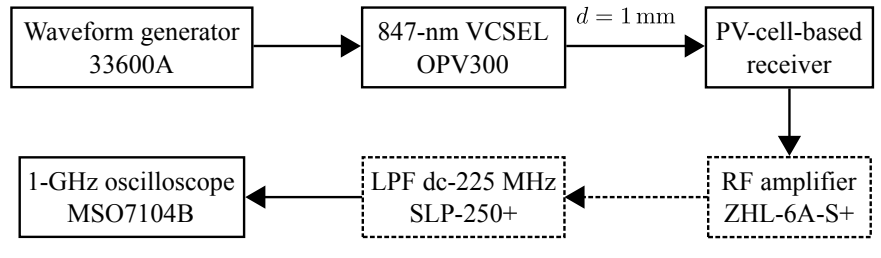

Fig. 1. System setup for the measurement of harvested power and frequency response.

1) Frequency response: An arbitrary waveform generator (AWG) $33600 \mathrm{~A}$ is used to create a sine wave of a peak-topeak (pp) voltage amplitude $v_{\mathrm{g}}=220 \mathrm{mV}$. The ac signal is added to a dc bias of $V_{\mathrm{dc}}=1.21 \mathrm{~V}$ used to drive a highspeed 847-nm VCSEL OPV300. The LPC is placed at a distance $d=1 \mathrm{~mm}$ from the laser diode (LD) and converts the optical signal to an electrical. As the load resistance is reduced, the mean pp voltage amplitude of the received ac signal decreases resulting in the increase of the quantization noise of the oscilloscope. Hence, a RF amplifier ZHL-6A-S+ is used to amplify the electrical power of the ac signal for $R_{1}=\{7.2 \Omega, 13.8 \Omega\}$ and maximize the available signal-toquantization-noise ratio. The RF amplifier consumes $7.1 \mathrm{~W}$ of de electrical power but it is used as an experimental instrument; significantly less power would be consumed by an amplifier optimized for real-world operation. The quantization noise of the oscilloscope is significantly limited by the use of higher $R_{1}$ values, e.g. $574 \Omega, 762.5 \Omega$ and $952 \Omega$, in parallel to its input $50-\Omega$ impedance; thus, the addition of an external amplifier provides negligible gain to the SNR and, therefore, is not required. The ac signal is then passed through a lowpass filter (LPF) SLP-250+ to reduce the noise. The ac voltage signal is captured and its mean pp amplitude is measured using a 1-GHz oscilloscope MSO7104B.

The frequency response can be defined by the normalized power gain $|H(f)|^{2}$ of the received ac voltage signal $v(t)$, where $f$ and $t$ denote frequency and time, respectively. The normalized power gain is calculated using $|H(f)|^{2}=$ $\left|v_{\mathrm{pp}}(f) / \max \left\{v_{\mathrm{pp}}(f)\right\}\right|^{2}$, where $v_{\mathrm{pp}}(f)$ is the mean pp voltage amplitude of $v(t)$. Let $\left|H_{\text {sys }}(f)\right|^{2}$ be used for the frequency response of the entire system shown in Fig. 1. The frequency response of the VCSEL $\left|H_{\mathrm{VCSEL}}(f)\right|^{2}$ is measured using the AWG, a photodiode-based receiver HSPR-X-I-1G4-SI with a 3-dB bandwidth of $1.4 \mathrm{GHz}$ and the oscilloscope. Also, the normalized power gain of the RF amplifier and the LPF $\left|H_{\text {amp }}(f)\right|^{2}$ is measured for an input pp voltage amplitude of $50 \mathrm{mV}$. Therefore, the frequency response of the PV cell $\left|H_{\mathrm{PV}}(f)\right|^{2}$ is determined using:

$$
\left|H_{\mathrm{PV}}(f)\right|^{2}=\left|\frac{H_{\mathrm{sys}}(f)}{H_{\mathrm{VCSEL}}(f) H_{\mathrm{amp}}(f)}\right|^{2} .
$$

2) Power transfer: A dc signal is created using the AWG with an offset $V_{\mathrm{dc}}=1.21 \mathrm{~V}$. The forward voltage of the LD is measured to be $V_{\mathrm{f}}=2.04 \mathrm{~V}$. The voltage of the diode is modified because its internal resistance is between $20 \Omega$ and $55 \Omega$, whereas an output load of $50 \Omega$ is expected by the AWG. The dc output optical power $P_{\mathrm{Tx}, \mathrm{opt}}$ of the VCSEL is measured using a $1-\mathrm{cm}^{2}$ sensor $\mathrm{S} 121 \mathrm{C}$ placed at a distance of 


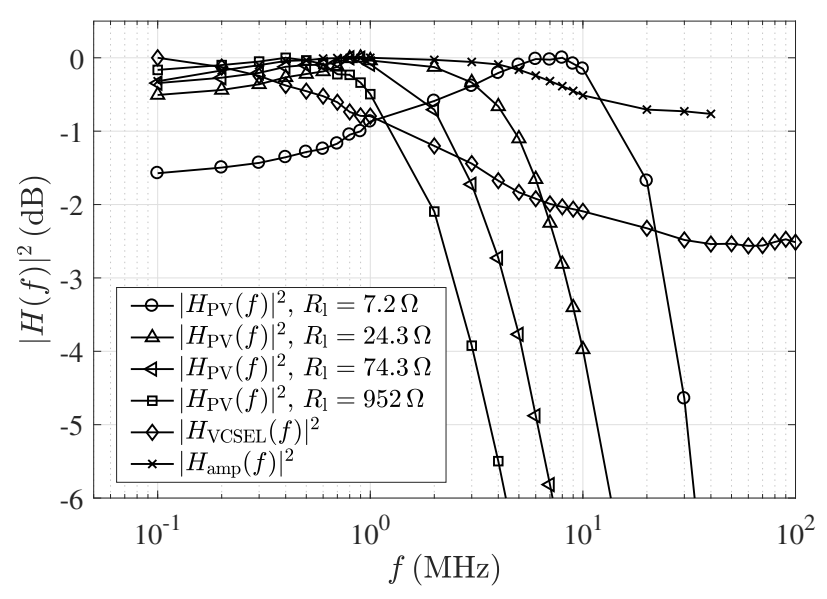

Fig. 2. Normalized power gain of the VCSEL, the RF amplifier with the LPF and the PV cell as a function of frequency.

less than $1 \mathrm{~mm}$ from the diode. Thus, the $847-\mathrm{nm}$ LD transmits a dc optical power $P_{\mathrm{Tx}, \mathrm{opt}}=3.62 \mathrm{~mW}$ at a forward voltage $V_{\mathrm{f}}=2.04 \mathrm{~V}$. This is the typical voltage-power point of the diode that is in the linear region of the stimulated emission. The PV efficiency $\eta_{\mathrm{PV}}$ of the cell is calculated by using $\eta_{\mathrm{PV}} \geq P_{\mathrm{Rx}, \mathrm{elec}} / P_{\mathrm{Tx}, \mathrm{opt}}$. According to a simulation of the optical setup in Zemax, 99.4\% of the LD's optical power is expected to be incident on the PV cell. Therefore, the optical intensity on the LPC is calculated to be $458 \mathrm{~mW} / \mathrm{cm}^{2}$ by using $G_{\mathrm{Rx}}=P_{\mathrm{Rx}, \mathrm{opt}} / A_{\mathrm{PV}}$, where $P_{\mathrm{Rx}, \mathrm{opt}}$ and $A_{\mathrm{PV}}$ denote the received optical power and area of the LPC, respectively.

\section{Results and discussion}

In Fig. 2, the normalized power gain of the VCSEL, the RF amplifier with the LPF and the PV cell is given as a function of frequency. The normalized power gain of the PV cell for $R_{1}=$ $7.2 \Omega$ is observed to be relatively small at low frequencies compared to that for $R_{1}=\{24.3 \Omega, 74.3 \Omega, 952 \Omega\}$. Thus, an inductive behavior of the LPC is observed at low resistance values due to the impedance mismatch between the receiver and the oscilloscope. The $3-\mathrm{dB}$ upper cut-off frequency of the $\mathrm{PV}$ cell for $R_{1}=7.2 \Omega$ is calculated to be $24.5 \mathrm{MHz}$ using linear interpolation between the two adjacent measured points $(20 \mathrm{MHz},-1.7 \mathrm{~dB})$ and $(30 \mathrm{MHz},-4.6 \mathrm{~dB})$. To the best of our knowledge, this is the largest reported value for PV cells used for data communication [3], [5], [6]. The addition of the RF amplifier after the LPC with the 7.2- $\Omega$ load offers a significant gain of $24.6 \mathrm{~dB}$ in the ac electrical power.

The 3-dB bandwidth and the power conversion efficiency of the PV cell are given as a function of the resistor load in Fig. 3. The experimental data of $\eta_{\mathrm{PV}}$ are fitted solving the optimization problem given in (1) under the conditions (2)(6). Table I summarizes the input parameters to the search algorithm along with the estimated parameters of the physical model of the PV cell. The objective function in (1) has a local minimum value of $1.1 \times 10^{-8} \mathrm{~A}^{2}$. The theoretical and experimental data of $\eta_{\mathrm{PV}}-R_{\mathrm{l}}$ are in close agreement with a mean squared error of $8.6 \times 10^{-6}$. The PV efficiency increases linearly with the increased load resistance reaching

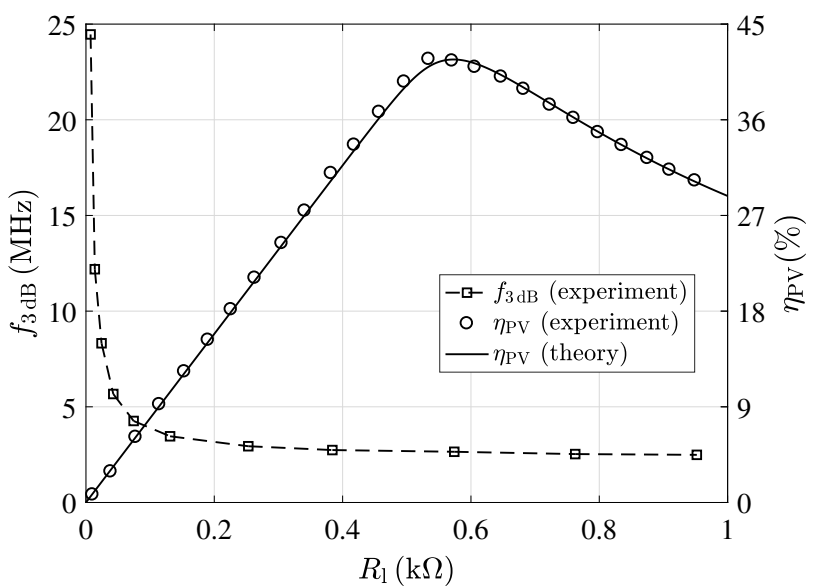

Fig. 3. Bandwidth of $3 \mathrm{~dB}$ and power efficiency of the PV cell as a function of load resistance.

its maximum point and, then, decreases exponentially; the highest power efficiency of the cell is measured to be $41.7 \%$ under the optical intensity of $0.46 \mathrm{~W} / \mathrm{cm}^{2}$ for $R_{1}=534 \Omega$. The 3-dB bandwidth of the PV-cell-based receiver is shown to decrease exponentially with an increase in load resistance. In the use case of a power-splitting double-branch-based receiver, the two functions of $\mathrm{EH}$ and data communication are shown to be optimized simultaneously [6]. In such a receiver, the $\mathrm{EH}$ branch should consist of a large inductor and a 534- $\Omega$ resistance. The data communication branch should comprise a large capacitor and a $7.2-\Omega$ resistor.

\section{DATA COMMUNICATION}

The experimental setup shown in Fig. 1 is used for the implementation of an OFDM-based OW link at a 1-mm distance. In addition, a laptop is connected to the AWG and the oscilloscope to perform the required digital-signal processing. As a next step, the distance of the $I R$ link is extended to $d=2 \mathrm{~m}$ using a plano-convex lens 86-921 and a condenser lens ACL4532-A at the transmitter and receiver, respectively.

\section{A. OFDM system}

The separate stages for the generation of an OFDM signal in MATLAB are pseudo-random data bit generation, adaptive bit and power loading, $M$-ary quadrature amplitude modulation (QAM), inverse fast Fourier transform (IFFT), oversampling, and pulse shaping [6]. The AWG is fed with the discrete OFDM signal and converts it to an analog waveform. The oscilloscope performs the analog-to-digital conversion of the received OFDM signal. The discrete signal is processed using synchronization, matched filtering, down-sampling, FFT, channel estimation, equalization, and $M$-ary quadrature amplitude demodulation in MATLAB [6]. To obtain a real OFDM signal after the IFFT operation, Hermitian symmetry needs to be applied to the second half of the OFDM frame [14]. Thus, the use of a FFT size of 1024 allows for the modulation of 511 subcarriers with unique data. The maximum QAM order $m$ is set to 1024 . The cyclic-prefix length is 5 ; and 
TABLE I

CURVE-FITTING PARAMETERS FOR PV EFFICIENCY

\begin{tabular}{llllllllllllll}
\hline $\begin{array}{l}I_{0, \min } \\
(\mathrm{fA})\end{array}$ & $\begin{array}{l}I_{0, \max } \\
(\mathrm{fA})\end{array}$ & $\begin{array}{l}R_{\mathrm{s}, \min } \\
(\Omega)\end{array}$ & $\begin{array}{l}R_{\mathrm{s}, \max } \\
(\Omega)\end{array}$ & $\begin{array}{l}n_{\min } \\
n_{\mathrm{max}}\end{array}$ & $\begin{array}{l}R_{\mathrm{sh}, \min } \\
(\mathrm{E} \Omega)\end{array}$ & $\begin{array}{l}R_{\mathrm{sh}, \max } \\
(\mathrm{E} \Omega)\end{array}$ & $\begin{array}{l}I_{\mathrm{ph}, \min } \\
(\mathrm{mA})\end{array}$ & $\begin{array}{l}I_{\mathrm{ph}, \max } \\
(\mathrm{mA})\end{array}$ & $\begin{array}{l}I_{0} \\
(\mathrm{fA})\end{array}$ & $\begin{array}{l}R_{\mathrm{s}} \\
(\Omega)\end{array}$ & $\begin{array}{l}n \\
-\end{array}$ & $\begin{array}{l}R_{\mathrm{sh}} \\
(\mathrm{E} \Omega)\end{array}$ & $\begin{array}{l}I_{\mathrm{ph}} \\
(\mathrm{mA})\end{array}$ \\
\hline 1 & 10 & 0.1 & 1 & 1 & 2 & 1 & 10 & 1.6 & 1.8 & 8.9 & 0.8 & 1.6 & 8 \\
\hline
\end{tabular}

TABLE II

DATA COMMUNICATION PARAMETERS

\begin{tabular}{lllr|lll}
\hline $\begin{array}{l}d \\
(\mathrm{~m})\end{array}$ & $\begin{array}{l}\varepsilon \\
(\mathrm{MHz})\end{array}$ & $\begin{array}{l}v_{\mathrm{g}} \\
(\mathrm{mV})\end{array}$ & $\begin{array}{l}R_{\mathrm{l}} \\
(\Omega)\end{array}$ & $\begin{array}{l}\xi \\
(\mathrm{dB})\end{array}$ & $\begin{array}{l}\omega \\
(\mathrm{Mb} / \mathrm{s})\end{array}$ & $\begin{array}{l}l \\
-\end{array}$ \\
\hline $10^{-3}$ & 62.5 & 400 & 7.3 & 29.0 & 505.2 & 2.2 \\
$10^{-3}$ & 62.5 & 400 & 574.0 & 13.8 & 202.1 & 0.9 \\
2 & 62.5 & 500 & 7.1 & 26.2 & 440.5 & 1.1 \\
2 & 125.0 & 500 & 7.1 & 15.5 & 522.1 & 1.3 \\
\hline
\end{tabular}

the minimum and maximum clipping levels of the timedomain OFDM signal are $-3.2 \sigma_{x}$ and $3.2 \sigma_{x}$, respectively where $\sigma_{x}$ is its standard deviation [6], [14]. A pilot sequence of 50 copies of the same OFDM frame is transmitted to the receiver for channel estimation. The estimated channel gain and variance of shot and thermal noise are used to determine the achievable SNR of each subcarrier. Thus, the constellation size of the QAM symbol that each subcarrier is modulated with is selected based on the SNR of that subcarrier. Frequency subcarriers that experience insufficient SNR values for data communication are not modulated.

\section{B. Results and discussion}

The average SNR of the subcarriers $\xi$, data rate $\omega$ and BER $b$ of the $847-\mathrm{nm}$ laser link are given in Table II. The input parameters are $d$, the single-sided communication bandwidth $\varepsilon, v_{\mathrm{g}}$, and $R_{\mathrm{l}}$. The load resistances are selected so that they are very close to the optimum values for data communication and $\mathrm{EH}$, respectively. A maximum spectral efficiency and SNR of $8.1 \mathrm{~b} / \mathrm{s} / \mathrm{Hz}$ and $29 \mathrm{~dB}$, respectively are achieved for $R_{\mathrm{l}}=7.3 \Omega$ and $v_{\mathrm{g}}=0.4 \mathrm{~V}$ at $d=1 \mathrm{~mm}$. The increase in load resistance from $7.3 \Omega$ to $574 \Omega$ results in a decrease in the SNR and data rate; this is attributed to the respective decrease in the $3-\mathrm{dB}$ bandwidth of the PV-cell-based receiver. The SNR and data rate for $R_{1}=7.3 \Omega$ increase significantly by $15.3 \mathrm{~dB}$ and $0.31 \mathrm{~Gb} / \mathrm{s}$ at $b=2.2 \times 10^{-3}$, respectively when the RF amplifier is used compared to its absence. However, whether the amplifier is used or not for $R_{1}=574 \Omega$, there is a trivial change in the SNR and data rate for a BER of $9 \times 10^{-4}$, i.e. $0.4 \mathrm{~dB}$ and $3.7 \mathrm{Mb} / \mathrm{s}$. This is because the noise induced by the oscilloscope is negligible compared to the amplified noise coming from the PV receiver. Doubling $\varepsilon$ from $62.5 \mathrm{MHz}$ to $125 \mathrm{MHz}$ at $d=2 \mathrm{~m}$ results in an increase in $\omega$ by 1.2 times, but the spectral efficiency and average SNR are reduced by $2.9 \mathrm{~b} / \mathrm{s} / \mathrm{Hz}$ and $10.7 \mathrm{~dB}$, respectively. Thus, a maximum data rate of $0.52 \mathrm{~Gb} / \mathrm{s}$ with a BER of $1.3 \times 10^{-3}$ is obtained at a 2-m link distance; this is the highest up-to-date value for OW communication and EH using PV cells.

\section{CONCLUSION}

In this letter, the highest up-to-date $3-\mathrm{dB}$ bandwidth of $24.5 \mathrm{MHz}$ was reported for PV cells; this is attributed to the reduced area and, therefore, capacitance of the GaAs LPC used compared with those of typical Si solar panels. For a receiver with a variable resistor, the data communication impairs exponentially with the increase in load resistance, while EH is constantly improved up to an optimum point. The harvested power decreases exponentially after its maximum value, whereas the data communication performance remains almost stable. The reported data rates of $0.5 \mathrm{~Gb} / \mathrm{s}$ using optimized bit-and-power-loaded OFDM pave the way for the deployment of free-space optical systems for SWIPT. Future work will consider the development of a PV receiver using two separate branches for optimal EH and data transfer.

\section{ACKNOWLEDGMENT}

The laser-link alignment at the 2-m distance along with relevant measurements was made with the help of Sovan Das.

\section{REFERENCES}

[1] I. Krikidis et al., "Simultaneous Wireless Information and Power Transfer in Modern Communication Systems," IEEE Commun. Mag., vol. 52, no. 11, pp. 104-110, Nov. 2014.

[2] X. Lu, P. Wang, D. Niyato, D. I. Kim, and Z. Han, "Wireless Networks With RF Energy Harvesting: A Contemporary Survey," Commun. Surveys Tuts., vol. 17, no. 2, pp. 757-789, Apr--June 2015.

[3] S.-M. Kim and J.-S. Won, "Simultaneous Reception of Visible Light Communication and Optical Energy Using a Solar Cell Receiver," in Proc. IEEE Int. Conf. ICT Convergence, Jeju Island, South Korea, Oct. 2013, pp. 896-897.

[4] Z. Wang, D. Tsonev, S. Videv, and H. Haas, "Towards Self-Powered Solar Panel Receiver for Optical Wireless Communication," in Proc. IEEE Int. Conf. Commun., Sydney, NSW, June 2014, pp. 3348-3353.

[5] S. Zhang et al., "Organic Solar Cells as High-Speed Data Detectors for Visible Light Communication," Optica, vol. 2, no. 7, pp. 607-610, July 2015.

[6] Z. Wang, D. Tsonev, S. Videv, and H. Haas, "On the Design of a SolarPanel Receiver for Optical Wireless Communications With Simultaneous Energy Harvesting," IEEE J. Sel. Areas Commun., vol. 33, no. 8, pp. 1612-1623, Aug. 2015.

[7] H. Helmers et al., "Integrated Power and Data Transceiver Devices for Power-by-Light Systems - A Concept Study," in 32nd Eur. Photovolt. Solar Energy Conf. Exhibition, Munich, Germany, June 2016, pp. 218 222.

[8] J. Fakidis, S. Videv, S. Kucera, H. Claussen, and H. Haas, "Indoor Optical Wireless Power Transfer to Small Cells at Nighttime," J. Lightw. Technol., vol. 34, no. 13, pp. 3236-3258, July 2016.

[9] J. L. Gray, "The Physics of the Solar Cell," in Handbook of Photovoltaic Science and Engineering, 2nd ed. New York, NY, USA: Wiley, June 2011, pp. 82-129.

[10] M. Pinuela, P. Mitcheson, and S. Lucyszyn, "Ambient RF Energy Harvesting in Urban and Semi-Urban Environments," IEEE Trans. Microw. Theory Techn., vol. 61, no. 7, pp. 2715-2726, July 2013.

[11] M. S. Islim et al., "The Impact of Solar Irradiance on Visible Light Communications," J. Lightw. Technol., to be published.

[12] O. Höhn, A. W. Walker, A. W. Bett, and H. Helmers, "Optimal Laser Wavelength for Efficient Laser Power Converter Operation over Temperature," Appl. Phys. Lett., vol. 108, no. 24, June 2016.

[13] W.-H. Shin, S.-H. Yang, D.-H. Kwon, and S.-K. Han, "Self-ReverseBiased Solar Panel Optical Receiver for Simultaneous Visible Light Communication and Energy Harvesting," Opt. Express, vol. 24, no. 22, pp. A1300-A1305, Oct. 2016.

[14] D. Tsonev et al., "A 3-Gb/s Single-LED OFDM-Based Wireless VLC Link Using a Gallium Nitride $\mu$ LED," IEEE Photon. Technol. Lett., vol. 26, no. 7, pp. 637-640, Apr. 2014. 